\title{
Entropy Method based Evaluation for Spectrum Usage Efficiency of International Mobile Telecommunication Systems*
}

\author{
Liqin Wang ${ }^{1}$, Tan Wang ${ }^{2}$, Zhaojun Qian² and Wei Li ${ }^{2}$. \\ ${ }^{1}$ North China Electric Power University, Beijing, 102206, China, 13391795680@163.com \\ 2 The State Radio Monitoring Center, Beijing, 100037, China \\ ** Correspoding author: The State Radio Monitoring Center, No.80 Xicheng Disrict, Beijing, 100037, \\ China. liwei@srrc.org.cn.
}

\begin{abstract}
Keywords: International Mobile Telecommunication (IMT) Systems, evaluating the usage of frequency bands, evaluation indicators
\end{abstract}

\begin{abstract}
Currently the radio spectrum resource has become increasingly scarce. Evaluating the usage of frequency bands with existing radio services, strengthening management of allocated resource and improving the spectrum utilization have been becoming an important way of improving spectrum management level. In particular, it is urgent to evaluate the efficiency of spectrum usage for International Mobile Telecommunication (IMT) Systems which is in great spectrum demand. In this paper, an evaluation indicator model is proposed according to the current situation of IMT Systems spectrum usage. The set of evaluation indicators is different from those of the conventional mobile communication network which is generally used for the operator network planning and network optimization. The study of evaluation model in this paper comes from spectrum manager's perspective. Firstly, this paper introduced investigation of spectrum management at home and aboard, then described the proposed evaluating indicators, finally we creatively combined entropy method with sample data based on the model .It can be seen that the proposed evaluation model is reasonable and helpful to support relevant spectrum management work.
\end{abstract}

\section{Introduction}

Since 1987, China's mobile communication has grown rapidly over the past thirty years which has achieved remarkable development both in scale and technology. In the end of 2015, the total number of China's mobile phone users reached to 1.306 billion, net improving 19.645 million compared with last year, the mobile phone user penetration ratio reached to 95.5\%; users' service has experienced from single voice service to the rapidly broadband data service. By 2015, mobile Internet access traffic consumption reached to 4.187 billion $\mathrm{G}$, improving $40.1 \%$ over last year, in which mobile Internet traffic reached 3.759 billion $\mathrm{G}$ [1].

The gradual development of commercial $4 \mathrm{G}$ and smart productions has greatly improved the efficiency of spectrum usage [2]. However, domestic and foreign research institutions show that in 2020, with the development of wireless technology, connecting hundreds of billions of devices and constantly increasing diverse business needs pose a serious challenge for us. the future of mobile communications service traffic will show explosive growth. So the evaluation of previous $2 \mathrm{G}, 3 \mathrm{G}$ and even 4G's spectrum usage efficiency will be great realistic significance.

Evaluation of the frequency bands utilization aims to reflect its status usage efficiency by establishing the indicator system of IMT. Comprehensive evaluation is widely applied in many fields, such as industry, agriculture, economy, Computer and so on which was firstly proposed from the article "test statistics" published in 1888 by Edgeworth [3]. From the angle of subjective weighting, people often use expert evaluation method, fuzzy comprehensive evaluation method, AHP [4] and so on. As for objective approaches, there are principal component analysis method, entropy method, neural network, TOPSIS method [5], etc. This paper innovatively uses the entropy method to processing data. 


\section{II.Research status at home and abroad}

Countries all attach great importance to managing spectrum resource because the spectrum resource is extremely valuable. They have done a large number of work for spectrum usage evaluation, spectrum management work of the typical regions is as follow:

The U. S. announced to work for spectrum resource inventory between 2011 to 2015 on April 13th 2010 by "RADIO Spectrum INVENTORY ACT", which provides inventory content: the radio services authorized to operate in each band of frequencies; the identity of each Federal or non-Federal user within each such radio service authorized to operate in each band of frequencies; the activities, capabilities, functions, or missions supported by the transmitters, end-user terminals or receivers etc [6].

The U.S. FCC released the "BROADBAND PROGRESS NOTICE OF INQUIRY" [7] report showing the initiative of evaluating today's fixed terrestrial broadband service-, and put mobile and satellite broadband service into the scope of broadband evaluation for the first time. This report claimed that the evaluating indicator must include speed, latency, consistent, and the data would be obtained from several authoritative laboratories such as Ookla, Rootmetrics and Google M-Lab for further implement of the evaluation.

The EU established a project group consisting of 27 member countries which is responsible for collecting the public and the dedicated spectrum applications at $400 \mathrm{MHz}-6 \mathrm{GHz}$ band. The team proposed a technology model and social benefit evaluation system of spectrum application to assess the technical efficiency of existing spectrum, they compared with other countries in the world for improving the usage efficiency of specific frequency bands.

British the earliest investigated mobile communication base station emissions and power level values in 2004 in the report "Mobile Phone Base-Station Audit"; British has a long-standing commitment to the audit of spectrum from 2002 with the aim of releasing the maximum amount of spectrum to the market and increasing opportunities for the development of innovative new services and completed "Independent Audit of Spectrum Holdings" in 2005[8]. Key recommendations include: the introduction of market mechanisms into spectrum management in the public sector; changes to the structure and scope of Administered Incentive Pricing as applied to the public sector; etc. In summary, countries all commit to spectrum management and spectrum monitoring work in long-term and the spectrum of the inventory is emphasized differently. Some countries focus on spectrum optimization, others focus on spectrum recycle. At present, there is not a country using the comprehensive evaluation method to evaluate the spectrum usage.

\section{III.Evaluation index}

Traditional measure of spectrum usage mainly through the spectrum occupancy, spectrum occupancy is the most direct way to reflect spectrum usage, within a period of time, if the received signal power strength is higher than the threshold value, which means the frequency band is occupied, or that is not occupied. In this paper, the evaluation indicator system is mainly considered from the three aspects: frequency, service, base stations, which makes up the deficiency of the existing single research method. The frequency includes the bandwidth and frequency occupied by the operator, and the service includes the rate of dropped calls, regional average telephone traffic and regional average data rate. The station includes the number of base stations within an unit area. The selection of the evaluation indicator is more comprehensive than the previous, and the correlation between the indicators is small. 
Table 1 Public mobile communication spectrum utilization efficiency evaluation index

\begin{tabular}{|c|c|c|c|}
\hline & Index & Formula & Unit \\
\hline Frequency & Bandwidth & Allocated Bandwidth & {$[\mathrm{MHz}]$} \\
\hline & User number & $\begin{array}{c}\text { The number of total user / } \\
\text { bandwidth }\end{array}$ & [number/MHz] \\
\hline & Frequency occupancy & devices measure & [percentage] \\
\hline Service & Drop rate & $\begin{array}{l}\text { Number of dropped calls / } \\
\text { Total number of calls }\end{array}$ & [percentage] \\
\hline & Regional average voice traffic & $\begin{array}{l}\text { Regional all day traffic/ } \\
\text { (Allocated Bandwidth*24) }\end{array}$ & {$\left[\mathrm{Erl} / \mathrm{MHz}^{*} \mathrm{~h}\right]$} \\
\hline & Regional average data rate & $\begin{array}{l}\text { Area throughput/ Allocated } \\
\text { Bandwidth }\end{array}$ & {$[\mathrm{Mbps} / \mathrm{MHz}]$} \\
\hline $\begin{array}{l}\text { Base } \\
\text { Station }\end{array}$ & Number of stations & $\begin{array}{l}\text { Number of base stations / } \\
\text { (9 square kilometers) }\end{array}$ & $\begin{array}{l}\text { [number/ } \\
\text { square } \\
\text { kilometre] }\end{array}$ \\
\hline
\end{tabular}

\section{IV.Evaluation method based on Entropy}

Mobile spectrum usage efficiency evaluation system has a number of indicators, how to determine the weights of these indicators is critical, the entropy method is a completely objective method for obtained the indicators weight, this paper will use entropy method to evaluate.

When there is a great difference between the data of one indicator, that less entropy, indicating that the large effective information content as well as large indicator weight; on the contrary, if a target sample data has a smaller difference, entropy will be bigger, which indicates the smaller amount of information and smaller weight. So according to the degree of difference between indicators achieving dynamic adjust the weight given by entropy.

With m objects, $\mathrm{n}$ evaluation indicators, the original indicator data matrix is obtained as follows:

$$
X=\left[\begin{array}{cccc}
x_{11} & x_{12} & \ldots & x_{1 m} \\
x_{21} & x_{22} & \ldots & x_{2 m} \\
\ldots & \ldots & \ldots & \ldots \\
x_{n 1} & x_{n 2} & \ldots & x_{4 m}
\end{array}\right]=\left(\mathrm{X}_{1}, \mathrm{X}_{2}, \ldots, \mathrm{X}_{\mathrm{m}}\right)
$$

If $X_{i}\{i=1,2,3 \ldots m\}$ is a negative indicator, that is, the smaller the value, the better indicators, We need to convert negative indicator to positive indicator as (2):

$X_{i}^{\prime}=1-X_{i}\{i=1,2,3 \ldots . m\}$

The proportion of the $i$ th sample under the $j$ th indicator is as:

$$
p_{i j}=\frac{x_{i j}^{\prime}}{\sum_{m}^{n} x_{i j}^{\prime}},(i=1,2, \ldots, n ; j=1,2, \ldots . m)
$$

The entropy the $j$ th indicator as (4):

$e_{j}=-k \sum_{i=1}^{n} p_{i j} \operatorname{In}\left(p_{i j}\right)$

Where: $k>0, k=1 / \operatorname{In}(n), e_{j} \geq 0$

The difference coefficient of the $j$ th indicator is like the formula (5).

$g_{j}=\frac{1-e_{j}}{m-E_{e}}, E_{e}=\sum_{j=1}^{m} e_{j}$

the weight of the $\mathrm{j}$ th indicator is as (6). 


$$
\omega_{i}=\frac{g_{i}}{\sum_{j=1}^{m} g_{i}}(1 \leq j \leq m)
$$

The sample score is (7):

$$
s_{i}=\sum_{j=1}^{m} \omega_{i} \bullet p_{i j},(i=1,2, \ldots n)
$$

\section{V.Instance verification}

Table 3 is a set of sample data obtained from the actual manufacturers, the data with entropy method obtained the weight of each indicator: $\mathrm{w}=(0.1587,0.1796,0.1201,0.1394,0.1360,0.1204$, 0.1457), and companies A, B, C evaluation scores respectively are 0.4023, 0.3285, 0.2692, thus draws the highest spectrum efficiency is manufacturer a, followed by manufacturer $\mathrm{B}$, spectrum efficiency lowest is manufacturer C.

Table 2 sample data

\begin{tabular}{llll}
\hline sample data & manufacturer A & manufacturer B & manufacturer C \\
\hline bandwidth & 140 & 60 & 70 \\
User number & $3.4 \mathrm{e} 8$ & $0.64 \mathrm{e} 8$ & $0.49 \mathrm{e} 8$ \\
Frequency occupancy & $96 \%$ & $98 \%$ & $99 \%$ \\
Drop rate & $2 \%$ & $2 \%$ & $3 \%$ \\
Average voice traffic areas & 4.46 & 5.56 & 4.76 \\
Regional average data rate & 100 & 80 & 60 \\
Base Station & $110 \mathrm{e} 4$ & $46 \mathrm{e} 4$ & $30 \mathrm{e} 4$ \\
\hline
\end{tabular}

\section{VI.Conclusion}

This paper considers that evaluating the usage efficiency of mobile communication evaluation should be a process from junior to senior in a long period of time. With the continuous improvement of testing equipment we can obtain more indicator data for more comprehensive evaluation; at the same time, we can also set indicator system from the economic, social benefits, environmental protection aspects etc by future more advanced means of signal identification and the analysis of big data for more scientific and effective evaluation. In this paper, we used entropy method to analysis data, in follow-up study we will consider using other methods showing the result of evaluation.

\section{Acknowledgements}

* This paper is supported by Chinese National Key Project under Grant No. 2015ZX03002008, and National HighTechnology Research and Development Program ("863Program) of China under Grant No. 2015AA01A705 and No.2014AA01A706.

\section{References}

[1] Information on http://www.miit.gov.cn

[2] Chen, Qian Zhang, Mingyan Liu, Shufang Li. Mining, in: Spectrum Usage Data: A Large-Scale Spectrum Measurement Study, IEEE Transactions on Mobile Computing (2012)

[3] T.J. Stewart, in: A critical survey on the status of multiple criteria decision, Omega (1991) 
[4] B. Michele, C. Christine, in: The analytic hierarchy process and the theory of measurement, Management Science (2010)

[5]Xu Xiaozhan, in: A note on the subjective and objective integrated approach to determine attribute weights, European Journal of Operational Research (2004)

[6] Information on https://www.ntia.doc.gov

[7] Information on https://www.fcc.gov/

[8]Information on https:http://www.spectrumaudit.org.uk 